\title{
Nanowire terahertz quantum cascade lasers
}

\author{
Thomas Grange* \\ Walter Schottky Institut, Technische Universität München, \\ Am Coulombwall 4, D-85748, Garching, Germany
}

(Dated: July 27, 2021)

\begin{abstract}
Quantum cascade lasers made of nanowire axial heterostructures are proposed. The dissipative quantum dynamics of their carriers is theoretically investigated using non-equilibrium Green functions. Their transport and gain properties are calculated for varying nanowire thickness, from the classical-wire regime to the quantum-wire regime. Our calculation shows that the lateral quantum confinement provided by the nanowires allows an increase of the maximum operation temperature and a strong reduction of the current density threshold compared to conventional terahertz quantum cascade lasers.
\end{abstract}

Quantum cascade lasers (QCLs) ${ }^{1}$ are semiconductor unipolar devices emitting coherent infrared radiation. Their active regions consist of stacked planar quantum wells (QWs) forming a superlattice along the growth direction. At first sight, a large part of the physical properties of QCLs can be qualitatively understood in a onedimensional picture, in particular for quantum confinement, tunneling processes and radiative transitions. Nevertheless, the free in-plane motion of the carriers provides a continuous energy reservoir (i.e. subbands) which plays a major role in the energy and phase relaxation processes. For QCLs operating in the terahertz $(\mathrm{THz})$ range $^{213}$, due to the relatively small energy separation between the subbands associated with the lasing levels, the non-radiative scattering processes are very efficient and are responsible for intrinsic limitations in terms of quantum efficiency and maximum operation temperature.

Reducing the dimensionality of electronic systems is generally a way to increase the carrier lifetimes 4 . 7 strong magnetic field applied along the growth axis of a QCL quantizes the lateral motion in terms of Landau levels. Their energy tuning with magnetic field has been shown to be responsible for modulation of the level lifetimes 89 , allowing to demonstrate lasing up to higher temperature $\left(225 \mathrm{~K}\right.$ in ref. $\left.{ }^{10}\right)$ than in usual $\mathrm{THz}$ QCLs (199 K in ref!11). However, Landau levels remain highly degenerate so that their ideal discrete density of states is strongly broadened by disorder effects ${ }^{12}$. On the other hand, QCLs based on 0D nanostructures such as self-assembled or lithographically defined quantum dots (QDs) have been proposed ${ }^{13}$ in order to achieve low threshold current and high operation temperature. However, a direct comparison of the device performances with respect to conventional THz QCLs is lacking, and the intermediate regime between the limiting cases coupled 0D QDs and coupled 2D QWs remains to be investigated.

In this letter we propose QCLs based on nanowire (NW) superlattice heterostructures. Using the formalism of non-equilibrium Green functions (NEGF), we calculate charge transport and $\mathrm{THz}$ gain in laterally-quantized quantum cascade (QC) structures. We investigate the dynamics of charge carriers in QC structures having lateral dimensionality ranging from $2 \mathrm{D}$ to $0 \mathrm{D}$, providing a direct comparison of NW QCLs and conventional QCLs.

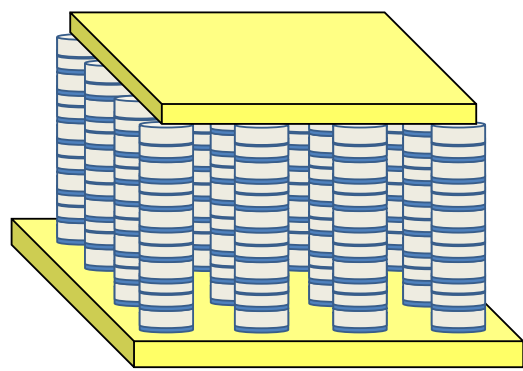

FIG. 1. Schematic of what a nanowire terahertz quantum cascade laser could look like. The active regions consists of nanowires containing axial periodic heterostructures. The space between the nanowires could be filled with a low-loss polymers 17 . The nanowire array could be sandwiched in a metal-metal waveguide.

Our calculations predict that below a critical thickness NW THz QCLs can achieve higher gain, higher operation temperature and lower current densities than conventional $\mathrm{THz}$ QCLs. For thin nanowires, large tunneling barriers in the axial heterostructure are shown to be necessary to avoid electrical instability owing to the increase of coherence lengths.

A schematic of the proposed NW-QCL is presented on Fig 1. The active region consists of an array of nanowires containing axial superlattice heterostructures. Experimentally, a micropillar version of such $\mathrm{THz}$ QCL geometry has been recently reported in 17 . We have recently studied theoretically electron transport in nanowire superlattices where one period consists of a single well and a single barrier ${ }^{18}$. The charge transport along the superlattice has been shown to depend dramatically on the nanowire lateral dimensions, with increasing coherence lengths predicted for decrasing nanowire thickness. The underlying reason is that lateral energy quantization greatly limits the scattering mechanisms allowed by energy conservation, resulting in longer lifetimes and coherence lengths.

We consider one of the nanowire of Fig 1 . The electronic structure is modeled within the effective mass approximation. The NW surface is assumed to be perfectly passivated so that no charge depletion or accumu- 
lation effects occur. We consider an electron wave function basis set of the form $\Psi_{\alpha n}=\psi_{\alpha}(z) \phi_{n}(\rho, \theta)$, where $\phi_{n}$ are the solutions of the Schrödinger equation on a homogeneous disc (ie the assumed NW cross-section) with eigenvalues $E_{n}$ and the $\psi_{\alpha}$ form a periodic basis set of the low energy states along the $z$ superlattice axis. The electron dynamics is calculated within the NEGF formalism in which the perturbative scattering processes are treated within the self-consistent Born approximation (SCBA). Self-consistent (SC) NEGF implementation of dissipative quantum transport in semiconducting heterostructures $\frac{18] 22}{22}$ allows a coherent and non-Markovian treatment of the charge dynamics, where the scattering self-energies can be conveniently treated on a microscopic basis. The SC-NEGF formalism has the advantage to correctly describe the intrinsic relation between scattering processes and line broadening effects, which is crucial here as we are interested in dimensionality transition and gain broadening.

We take into account the scattering mechanisms that are known to be important in QW QCLs heterostructures: longitudinal optical (LO) and acoustical phonon emission/absorption, interface roughness, charged impurities and alloy disorder. Electron-electron scattering is not considered. It is expected to play a minor role in the resonant-phonon Thz QCL design studied below 35 . In addition, scattering mechanisms specific to NWs and/or quasi 0D systems are also considered. The NW surface roughness is taken into account as an additional elastic scattering mechanism. NW phonon confinement effects are considered and we calculate the coupling of electrons to longitudinal optical (LO) and surface optical (SO) phonons, as well as confined acoustic phonon $\$ 18$.

In quasi 0D systems, early studies have predicted a phonon bottleneck as soon as discrete electronic transitions are detuned from the optical or acoustical phonon frequencies ${ }^{424}$. More recent studies have demonstrated that (i) direct LO-phonon emission/absorption processes are totally suppressed even in case of electron-LOphonon resonances due to the strong coupling regime between electrons and LO-phonons, and that (ii) the dominant energy relaxation of terahertz excitations is the polaron anharmonicity, i.e. an indirect scattering process involving electron-LO-phonon polar interaction as well as anharmonic couplings of LO-phonons with other phonon modes 51726 . This mechanism is included here, beyond a phenomenological broadening of the optical phonon GFs 21 , by calculating the phononic self-energies of the optical phonons due to their anharmonic coupling with the other phonon modes 18 .

The elastic scattering mechanisms induced by disorder effects (here interface roughness, surface roughness, alloy disorder and charged impurities) are usually treated within the SCBA in planar QW heterostructures $\frac{\sqrt{19|20| 22} \text {. }}{\text {. }}$ However, the SCBA only accounts for an incoherent treatment of the disorder effects. Here, as the NW gets thinner, the elastic scattering becomes mainly coherent, as the disorder potential fluctuations become larger than
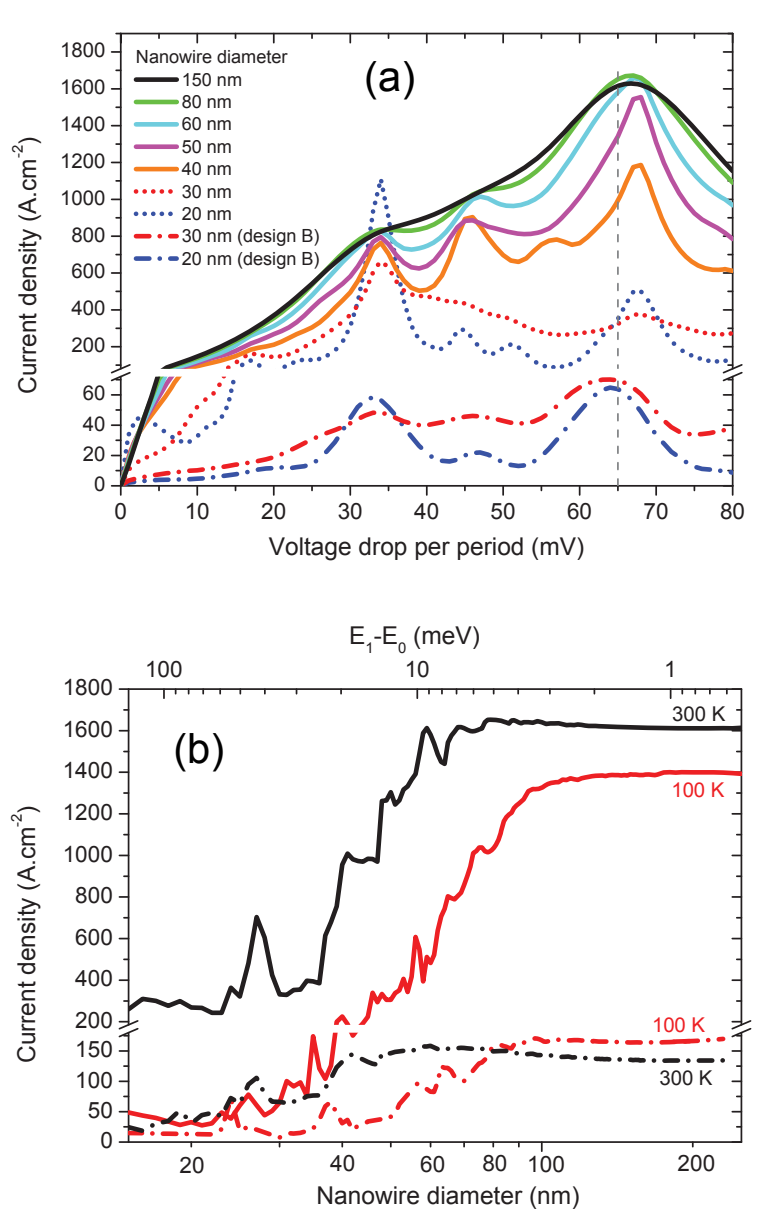

FIG. 2. Transport properties of NW QCLs. Axial designs $A, B_{300}$ and $B_{100}$ are studied with layer thickness of respectively 14/1.5/11.2/3.5/22/3.5, 14.4/1.5/11.5/7/23/7 and 14.5/1.5/11.6/7/22.5/7 (barriers are in bold fonts and doped layer underlined). (a) Current density as a function of the voltage drop per period for various NW diameters at lattice temperature of $300 \mathrm{~K}$, for the design A (solid and dotted lines for respectively stable and unstable operating regions) and for the design $\mathrm{B}_{300}$ (dashed-dotted lines). The designed operating bias $(65 \mathrm{mV})$ is indicated by a vertical dotted line. (b) Current density as a function of the NW diameter for fixed operating bias voltage at lattice temperatures of $100 \mathrm{~K}$ (red lines) and $300 \mathrm{~K}$ (black lines) for the design $\mathrm{A}$ in solid lines and the designs $\mathrm{B}$ in dashed-dotted lines $\left(\mathrm{B}_{100}\right.$ at $100 \mathrm{~K}$ and $\mathrm{B}_{300}$ at $\left.300 \mathrm{~K}\right)$. The top axis indicates the energy separation between the NW lateral ground state $\phi_{0}$ and the first excited state $\phi_{1}$.

the phonon-limited broadening. In the present work, a coherent treatment of the disorder potential is made as reported in Ref. $[18$.

We consider here the $\operatorname{In}_{0.53} \mathrm{Ga}_{0.47} \mathrm{As} / \mathrm{GaAs}_{0.51} \mathrm{Sb}_{0.49}$ material system lattice matched to InP, in which a conventional THz QCL operating up to $142 \mathrm{~K}$ has recently been demonstrated 27 . This system could be used to realize NW QCLs in a top-down approach 17 , while bottomup approaches might be envisaged on the longer term 
with similar material systems 28129 . We consider below 3-well resonant phonon designs (A and $\mathrm{B}$ ), similar to the one holding operation temperature records in conventional $\mathrm{THz}$ QCLs11. The layer sequence in nanometers of the design $\mathrm{A}$ is $14 / \mathbf{1 . 5} / 11.2 / \mathbf{3 . 5} / \underline{22} / \mathbf{3 . 5}$ with barriers indicated in bold fonts and the doped layer underlined. The equivalent areal doping density per period is set to $3 \times 10^{10} \mathrm{~cm}^{-2}$. The interface and surface roughnesses are both assumed to have a rms fluctuating amplitude of $3 \AA$ with correlation lengths of $8 \mathrm{~nm}$. The filling factor of the NW array is assumed to be unity for simplicity in the treatment of Coulombic interactions and for clear comparison with the conventional QCL geometry. All the remaining parameters in our model are material-dependent and we take standard values from the literature $30 \mid 31$.

Fig. 2 shows the calculated transport properties of the NW QCLs. The current-voltage characteristics calculated for various NW diameters at room temperature are shown on Fig. [a. Fig. [p shows the current density as a function of the NW diameter for a fixed applied bias of $65 \mathrm{mV}$ per period for various temperatures. For sufficiently thick wires the calculated current densities saturate on Fig. [b and all the IV curves for diameters larger than $\sim 150 \mathrm{~nm}$ (not shown on Fig. (a) overlap. This corresponds to the $2 \mathrm{D}$ regime of the lateral motion, i.e. when the lateral quantization effects are negligible. When the NW diameter is reduced below a temperature-dependent critical diameter, variations of the current density are observed on Fig. [b corresponding to the appearance of lateral dimensional effects. In this quantum wire regime, the $\mathrm{I}-\mathrm{V}$ characteristic possesses more distinct peaks as the reduction of the scattering processes allows adiabatic transport over larger distances. In fact, in the $2 \mathrm{D}$ regime the lateral motion provides an energy reservoir towards which the excess axial energy is easily transfered via the disorder potentials. As the lateral motion gets quantized, this elastic energy exchange tends to be suppressed $\frac{18}{10}$. As the remaining scattering processes are mainly due to optical phonons, a strong current peak remains for potential drop per period matching the optical phonon energy (34 $\mathrm{meV}$ ), whereas the current is strongly reduced for other bias voltage. For thin diameters, as this parasitic current peak at $34 \mathrm{mV}$ becomes larger than the current at the laser operating bias (around $65 \mathrm{meV}$ ), electrical instability can occur in the design A. In order to restore electrical stability, designs B differing from the design A mainly by twice thicker injector and extractor tunneling barriers (7 $\mathrm{nm}$ instead of $3.5 \mathrm{~nm}$ ) are investigated. Owing to the sharper tunneling resonances and the effective mass temperature dependence, two slighty different designs $B_{300}$ $\left(\mathrm{B}_{100}\right)$ with layer sequence in $\mathrm{nm} 14.4 / \mathbf{1 . 5} / 11.5 / \mathbf{7} / \underline{23} / \mathbf{7}$ $(14.5 / \mathbf{1 . 5} / 11.6 / \mathbf{7} / \underline{22.5} / \mathbf{7})$ are studied at respectively 300 $\mathrm{K}$ and $100 \mathrm{~K}$. As shown on Fig. [a at $300 \mathrm{~K}$, the weaker tunneling couplings in designs $\mathrm{B}$ allow a reduction of the current leakage peak at the optical phonon energy. A further insight into the nature of transport is provided by the analysis of the spectral function (not shown here). For the thick injector and collector barriers in the design

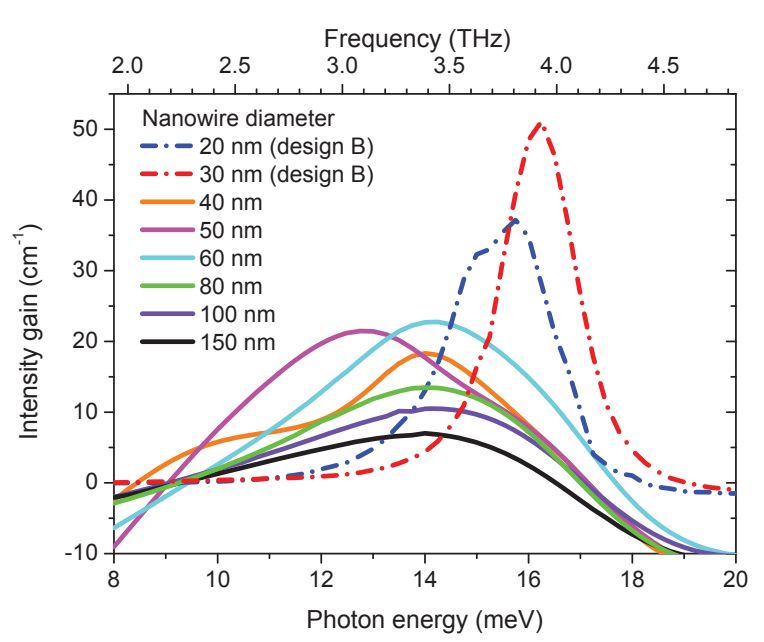

FIG. 3. Intensity gain spectra at $300 \mathrm{~K}$ calculated for various NW diameter for the design A (solid lines) and the design $\mathrm{B}_{300}$ (dashed-dotted lines).

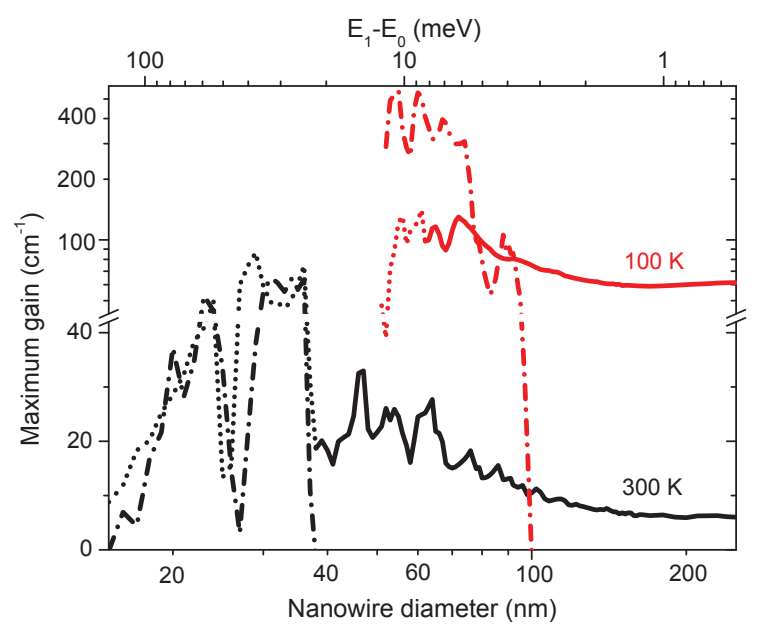

FIG. 4. Maximum intensity gain as a function of the NW diameter at $300 \mathrm{~K}$ (black lines) and $100 \mathrm{~K}$ (red lines) for the design A (solid and dotted lines for respectively electrical stable and unstable gain regions) and for the designs B (dasheddotted lines, only for NW diameters satisfying electrically stability).

A, anticrossings in the spectral function at the tunneling resonances are observed only for NW diameters smaller than $\sim 35 \mathrm{~nm}(\sim 60 \mathrm{~nm})$ at $300 \mathrm{~K}(100 \mathrm{~K}$ respectively $)$, indicating than the nature of these resonant tunneling processes evolves from an incoherent to a coherent regime as the NW thickness is reduced.

We calculate the $\mathrm{THz}$ gain in linear response with a self-consistent treatment of the induced self-energies (i.e. including vertex corrections $)^{32}$, which has been shown to be necessary to correctly treat the correlation in the scattering potentials 33 . The calculated intensity gain spectra at the optimal operating bias are shown on Fig. 3 at 
$300 \mathrm{~K}$. The peak values of the gain are plotted on Fig. 4 as a function of the NW diameter at $100 \mathrm{~K}$ and $300 \mathrm{~K}$. The gain of the design A in the QW limit reaches $6 \mathrm{~cm}^{-1}$ at room temperature, which is below the typical reported cavity losses 34 . As the NW diameter decreases into the quantum wire regime, the gain tends to increase. This increase is attributed to the overall decrease of scattering mechanism efficiency with decreasing dimensionality, and hence increase in the lifetimes and coherence times. Peaks in the gain are observed for certain NW diameters on Fig. 4. This is due to the interplay between the energy of the axial heterostructure and the lateral energy quantization, the former being tuned by the NW diameter while the first is fixed. A narrowing of the gain peaks with decreasing NW diameter is also observed on Fig. 3. This is attributed to a decrease of the scattering process efficiency and consequently of the homogeneous transition linewiths. Inhomogeneous broadening effects are found to remain smaller than homogeneous ones for NW diameters down to $20 \mathrm{~nm}$. Fig. 4 allows to predict the required NW diameters in order to obtain lasing. Assuming cavity losses equivalent to an absorption of $15(30) \mathrm{cm}^{-1}$, it shows that $\mathrm{THz}$ lasing at $300 \mathrm{~K}$ requires $\mathrm{NW}$ diameters smaller than $70(38) \mathrm{nm}$. As the NW diameter is further reduced below $38 \mathrm{~nm}$ at $300 \mathrm{~K}(62 \mathrm{~nm}$ at $100 \mathrm{~K})$, the design A becomes electrically unstable (dotted lines on Fig. (4). The design B is stable down to thinner NW diameters and its gain for NW diameter between 30 and 36 $\mathrm{nm}$ reaches values around $60 \mathrm{~cm}^{-1}$ at room temperature, which is equivalent to the calculated value at $100 \mathrm{~K}$ in the $2 \mathrm{D}$ limit. For NW diameters below $20 \mathrm{~nm}$, inhomogeneous energy fluctuations start to dominate over homogeneous broadening, so that the gain strongly decreases due to the large energy fluctuations and the consequent misalignment of the QCL levels. The calculated gain for the designs $\mathrm{B}\left(\mathrm{B}_{300}\right.$ and $\left.\mathrm{B}_{100}\right)$ is found to be more sensitive to the NW diameter with respect to design $\mathrm{A}$ : it is positive below a critical diameter $(38 \mathrm{~nm}$ at $300 \mathrm{~K}$ and $100 \mathrm{~nm}$ at $100 \mathrm{~K}$ ), and negative beyond up to the $2 \mathrm{D}$ limit. Indeed, coherent propagation through their large injector and collector tunneling barriers occurs only in the quantum wire regime, whereas in the $2 \mathrm{D}$ limit the tunneling is mainly incoherent and not efficient enough to allow population inversion. A further advantage of the designs $\mathrm{B}_{300}$ and $\mathrm{B}_{100}$ in the quantum wire regime resides in their much smaller current densities threshold (more than one order of magnitude smaller) than the design A operating in the $2 \mathrm{D}$ limit.

In summary, we have predicted the transport properties of NW QC structures as well as their $\mathrm{THz}$ response. We have shown that NWs QC structures allow higher $\mathrm{THz}$ gain and together with lower current densities compared to conventional planar QCs structures. Large tunneling barriers are shown to be necessary in the thin NW regime in order to maintain electrical stability as coherent propagation lengths are increased. Finally, the present work provides the geometrical requirements of NW QC structures in order to achieve $\mathrm{THz}$ stimulated emission at room temperature.

This work has been supported by the Alexander von Humboldt foundation and the Austrian Science Fund FWF (SFB IR-ON). S. Birner, M. Branstetter, C. Deutsch, P. Greck, G. Koblmüller, M. Krall, T. Kubis, S. Rotter, K. Unterrainer and P. Vogl are gratefully acknowledged for fruitful discussions.
* thomas.grange@neel.cnrs.fr Present address: Institut Néel-CNRS, 25 av. des Martyrs, 38042 Grenoble, France.

1 J. Faist, F. Capasso, D. L. Sivco, C. Sirtori, A. L. Hutchinson, and A. Y. Cho, Science 264, 553 (1994).

2 R. Kohler, A. Tredicucci, F. Beltram, H. E. Beere, E. H. Linfield, A. G. Davies, D. A. Ritchie, R. C. Iotti, and F. Rossi, Nature 417, 156 (2002).

3 B. S. Williams, Nat Photon 1, 517 (2007).

${ }^{4}$ U. Bockelmann and G. Bastard, Phys. Rev. B 42, 8947 (1990).

5 S. Sauvage, P. Boucaud, R. P. S. M. Lobo, F. Bras, G. Fishman, R. Prazeres, F. Glotin, J. M. Ortega, and J.-M. Gérard, Phys. Rev. Lett. 88, 177402 (2002).

6 A. Pandey and P. Guyot-Sionnest, Science 322, 929 (2008).

7 E. Zibik, T. Grange, B. Carpenter, N. Porter, R. Ferreira, G. Bastard, D. Stehr, S. Winnerl, M. Helm, H. Liu, et al., Nature materials 8, 803 (2009).

8 C. Becker, C. Sirtori, O. Drachenko, V. Rylkov, D. Smirnov, and J. Leotin, Applied physics letters 81, 2941 (2002).

9 C. Becker, A. Vasanelli, C. Sirtori, and G. Bastard, Physical Review B 69, 115328 (2004).

10 A. Wade, G. Fedorov, D. Smirnov, S. Kumar, B. Williams,
Q. Hu, and J. Reno, Nature Photonics 3, 41 (2008).

11 S. Fathololoumi, E. Dupont, C. Chan, Z. Wasilewski, S. Laframboise, D. Ban, A. Mátyás, C. Jirauschek, Q. Hu, and H. Liu, Optics Express 20, 3866 (2012).

12 A. Leuliet, A. Vasanelli, A. Wade, G. Fedorov, D. Smirnov, G. Bastard, and C. Sirtori, Physical Review B 73, 085311 (2006).

13 N. Wingreen and C. Stafford, Quantum Electronics, IEEE Journal of 33, 1170 (1997).

14 C. Hsu, O. Jeong-Seok, P. Zory, and D. Botez, Selected Topics in Quantum Electronics, IEEE Journal of 6, 491 (2000).

15 I. A. Dmitriev and R. A. Suris, physica status solidi (a) 202, 987 (2005).

16 N. Vukmirovic, D. Indjin, Z. Ikonic, and P. Harrison, Photonics Technology Letters, IEEE 20, 129 (2008).

17 M. Krall, M. Brandstetter, C. Deutsch, H. Detz, A. M. Andrews, W. Schrenk, G. Strasser, and K. Unterrainer, Optics express 22, 274 (2014).

18 T. Grange, Phys. Rev. B 89, 165310 (2014).

19 R. Lake, G. Klimeck, R. C. Bowen, and D. Jovanovic, Journal of Applied Physics 81, 7845 (1997).

20 S.-C. Lee and A. Wacker, Phys. Rev. B 66, 245314 (2002). 
${ }^{21}$ N. Vukmirovic, Z. Ikonic, D. Indjin, and P. Harrison, Phys. Rev. B 76, 245313 (2007).

22 T. Kubis, C. Yeh, P. Vogl, A. Benz, G. Fasching, and C. Deutsch, Phys. Rev. B 79, 195323 (2009).

${ }^{23}$ H. Xie, C. Chen, and B. Ma, Physical Review B 61, 4827 (2000).

24 H. Benisty, C. M. Sotomayor-Torrès, and C. Weisbuch, Phys. Rev. B 44, 10945 (1991).

25 S. Hameau, Y. Guldner, O. Verzelen, R. Ferreira, G. Bastard, J. Zeman, A. Lemaître, and J. M. Gérard, Phys. Rev. Lett. 83, 4152 (1999).

26 T. Grange, R. Ferreira, and G. Bastard, Phys. Rev. B 76, R241304 (2007).

27 C. Deutsch, M. Krall, M. Brandstetter, H. Detz, A. M. Andrews, P. Klang, W. Schrenk, G. Strasser, and K. Unterrainer, Applied Physics Letters 101, 211117 (pages 4) (2012).

28 L. Samuelson, C. Thelander, M. Björk, M. Borgström, K. Deppert, K. Dick, A. Hansen, T. Mårtensson, N. Panev,
A. Persson, et al., Physica E: Low-dimensional Systems and Nanostructures 25, 313 (2004).

29 P. Krogstrup, J. Yamasaki, C. Sørensen, E. Johnson, J. Wagner, R. Pennington, M. Aagesen, N. Tanaka, and J. Nygård, Nano letters 9, 3689 (2009).

30 I. Vurgaftman, J. Meyer, and L. Ram-Mohan, Journal of applied physics 89, 5815 (2001).

31 M. Levinshtein, M. S. Shur, and S. Rumyanstev, Handbook series on semiconductor parameters, v. 2 (World Scientific, 1996).

32 A. Wacker, Physical Review B 66, 085326 (2002).

33 F. Banit, S. Lee, A. Knorr, and A. Wacker, Applied Physics Letters 86, 041108 (2005).

34 D. Burghoff, C. W. I. Chan, Q. Hu, and J. L. Reno, Applied Physics Letters 100, 261111 (pages 4) (2012).

35 C. Jirauschek and P. Lugli, Journal of Applied Physics 105, 123102 (2009). 